\title{
Evaluación de la fatiga en pacientes con artritis psoriásica y su asociación con otras variables de la enfermedad
}

\author{
J. Gallino Yanzi ${ }^{1}$, O. Cerda ${ }^{1}$, C.A. Zaffarana ${ }^{1}$, M. Landi ${ }^{1}$, E.E. Schneeberger ${ }^{1}$, I. Carrillo', M.C. González Guzmán ${ }^{1}$, \\ M.P. Kohan², E. Buschiazzo ${ }^{3}$, J.A. Maldonado Cocco', G. Citera1 \\ ${ }^{1}$ Instituto de Rehabilitación Psicofísica, CABA, Argentina, ${ }^{2}$ Hospital Dr. E. Tornú, CABA, Argentina, ${ }^{3}$ Hospital Señor del Milagro, Salta, \\ Argentina.
}

\section{Resumen}

Objetivo: Evaluar la frecuencia de fatiga en pacientes con APs y su asociación con otras variables de la enfermedad.

Métodos: Pacientes $\geq 18$ años con diagnóstico de APs según criterios CASPAR. Se evaluó fatiga, rigidez matinal, dolor, actividad de la enfermedad por el paciente y el médico por escala visual graduada (EVG). Se completaron los autocuestionarios ASQoL, PSAQoL, HAQ-A, BASFI y BASDAl. Se calcularon los índices compuestos DAS28, DAPSA y CPDAI.

Resultados: Se incluyeron 112 pacientes. La mediana de fatiga fue de $3 \mathrm{~cm}$ (RIC 0-6). Fatiga tuvo aceptable correlación con BASDAl $(r: 0,63)$, pregunta $N^{0} 1$ del BASDAl $(r: 0,48)$, DAS28 $(r: 0,45)$, DAPSA (r:0,5), HAQ-A (r:0,42), PSAQoL (r:0,49), ASQoL (r:0,48), BASFI ( $r: 0,47)$, EVG de dolor $(r: 0,48)$ y de rigidez matinal $(r: 0,55)$. El $30 \%$ de nuestra cohorte presentó fatiga definida como EVG $\geq 6$ $\mathrm{cm}$. En el análisis de regresión lineal múltiple, la actividad de la enfermedad articular periférica (DAPSA coef $\beta: 0,36, p=0,0001$ ) y la calidad de vida (PsAQoL coef $\beta: 0,27, p=0,009$ ) se asociaron significativamente a fatiga.

Conclusión: El 30\% de nuestra cohorte de pacientes presentó EVG de fatiga $\geq 6 \mathrm{~cm}$. La fatiga se asoció independientemente con la actividad de la enfermedad articular periférica y la calidad de vida.

Palabras clave: fatiga, artritis psoriásica.

\section{Abstract}

Objective: To evaluate the frequency of fatigue in patients with PsA and its association with other disease variables.

Methods: We included patients $\geq 18$ years of age with PsA according to CASPAR criteria. Fatigue, morning stiffness, pain and global activity by both patients and physicians were assessed using visual analogue scales (VAS). ASQoL, PSAQoL, HAQ-A, BASFI and BASDAI were completed. DAS28, DAPSA and CPDAl were calculated.

Results: We included 112 patients. Median fatigue VAS was 3 $\mathrm{cm}$ (IQR 0-6). Fatigue had an acceptable correlation with BASDAI $(r: 0.63)$, BASDAl question $N^{0} 1$ ( $\left.r: 0.48\right)$, DAS28 ( $\left.r: 0.45\right)$, DAPSA (Rho:0.5), HAQ-A (Rho:0.42), PsAQol (Rho:0.49), ASQoL (Rho:0.48), BASFI (Rho:0.47), pain (Rho:0.48) and morning stiffness (Rho:0.55) and a regular correlation with CPDAl (Rho:034). $30 \%$ of our cohort had fatigue, as defined by a VAS equal or greater than $6 \mathrm{~cm}$. In multiple linear regression analysis, adjusting for sex, age and disease duration, peripheral disease activity (DAPSA $\beta$ coeff: 0.36 , $p=0.0001$ ) and quality of life (PSAQoL $\beta$ coeff: $0.27, p=0.009$ ) were associated with fatigue.

Conclusion: Prevalence of fatigue in this cohort was $30 \%$ and it was associated with greater peripheral disease activity and worse quality of life.

Key words: fatigue, psoriatic arthritis.

\section{Correspondencia}

E-mail: gustavocitera@gmail.com 


\section{Introducción}

La Artritis Psoriásica (APs) es una artropatía inflamatoria que se presenta en el 5-40\% de los pacientes con psoriasis cutánea ${ }^{1}$. En nuestro país, la incidencia de esta patología es de 6,26 casos nuevos cada 100.000 habitantes/año ${ }^{2}$. Afecta a personas de ambos sexos, más frecuentemente entre los 20 y 40 años. El cuadro es variado e incluye compromiso del esqueleto axial, oligoartritis asimétrica o poliartritis, dactilitis y entesitis, pudiendo presentar también síntomas generales como fatiga, anorexia o pérdida de peso $^{3}$. La APs se asocia a discapacidad funcional, disminución de los años de vida productivos y deterioro de la calidad de vida, con un impacto en la esfera personal, familiar y social del paciente y aumento de la mortalidad ${ }^{4,5}$. En varios estudios se concluyó que la carga de esta enfermedad es similar a la de pacientes con Artritis Reumatoidea (AR $)^{6,7}$ o Espondilitis Anquilosante (EA) ${ }^{7}$. Rosen y cols. demostraron que la calidad de vida de pacientes con APs es peor que la de aquellos con psoriasis cutánea ${ }^{8}$.

Una combinación de factores como el dolor, la discapacidad funcional y los trastornos psicológicos, están asociados a la presencia de fatiga en pacientes con $\mathrm{APs}^{10}$. El objetivo del presente estudio fue evaluar la frecuencia de fatiga en pacientes con APs y su asociación con otras variables de la enfermedad.

\section{Materiales y métodos}

Realizamos un estudio multicéntrico, de corte transversal, incluyendo pacientes de ambos sexos, $\geq 18$ años con diagnóstico de APs según criterios CASPAR ${ }^{11}$. Se excluyeron aquellos pacientes que por algún impedimento físico o psíquico no pudieron realizar los cuestionarios (analfabetos, ciegos), aquellos con diagnóstico de APs antes de los 16 años y los que cumplían criterios ACR para fibromialgia ${ }^{12}$, en este último caso debido a que la fatiga podría estar relacionada e influenciada por esta patología. Se recolectaron datos sociodemográficos, como sexo, edad, estado civil, escolaridad, ocupación actual, cobertura social, presencia de comorbilidades y tratamiento actual. En el examen físico se evaluó el número de articulaciones dolorosas (68) y tumefactas (66), se evidenciaron las zonas de entesitis por medio del MASES (Maastricht Ankylosing Spondylitis Enthesitis Score $)^{13}$. La Psoriasis cutánea se valoró por medio del PASI (Psoriasis Area Severity Index) $)^{14}$, compromiso ungueal por el PNSS (Psoriasis Nail Severity Score) ${ }^{15}$ y movilidad axial por BASMI (Bath Ankylosing Spondylitis Metrology Index $)^{16}$. Se consignaron reactantes de fase aguda Proteína C Reactiva (PCR mg/dl) y eritrosedimentación (ERS $\mathrm{mm} / \mathrm{h}$ ). Se evaluaron rigidez matinal, dolor, actividad de la enfermedad por el paciente y el médico y fatiga por medio de Escala Visual Graduada (EVG 0-10 centímetros). Se realizaron autocuestionarios para evaluar calidad de vida ASQoL (Ankylosing Spondylitis Quality of Life) ${ }^{17}$, PsAQoL (Psoriatic Arthritis Quality of Life) ${ }^{18}$ y DLQI (Dermatology Life Quality Index $)^{19}$, capacidad funcional HAQ-A (Health Assessment Questionnaire versión Argentina) ${ }^{20}$ y BASFI (Bath Ankylosing Spondylitis Functional Index $)^{21}$ y actividad de la enfermedad a través de BASDAI (Bath Ankylosing Spondylitis Disease Activity Index ${ }^{22}$. Se calcularon los índices compuestos DAS28 (Disease Activity Score) ${ }^{23}$, DAPSA (Disease Activity for Psoriatic Arthritis) ${ }^{24}$, CPDAI (Composite Psoriatic Disease Activity Index) ${ }^{25}$, SDAI (Simplifie Disease Activity Index ${ }^{26}$, CDAI (Clinical Disease Activity Index) ${ }^{27}$ e IAS (Índice de Actividad Simplificado) ${ }^{28}$ para evaluar actividad periférica.

Análisis estadístico: Las variables categóricas se expresaron en frecuencia y porcentaje, y las continuas en mediana $(m)$ y rango intercuartilo (RIC). Para comparar variables continuas utilizamos test de T Student y ANOVA y para las variables categóricas test de $\mathrm{Chi}^{2}$ y test exacto de Fisher. Correlaciones por test de Spearman. Análisis de regresión lineal múltiple utilizando como variable dependiente la fatiga por EVG, aquellas variables asociadas con fatiga en el análisis univariado $(\mathrm{p}<0,1)$ fueron incluidas como variables independientes en el análisis multivariado. Una $\mathrm{p}<0,05$ fue considerada significativa.

\section{Resultados}

Se incluyeron 112 pacientes, 57 eran varones (50,9\%), con una edad mediana de 54 años (RIC 42-63) y una duración mediana de la enfermedad de 9 años (RIC 5-15). 43 pacientes $(38,4 \%)$ presentaban compromiso mixto, 35 oligoarticular (31,2\%), 29 poliarticular $(25,9 \%), 3$ axial puro $(2,7 \%)$ y 2 mutilante $(1,8 \%)$. En cuanto al tratamiento, 79 pacientes $(70,4 \%)$ recibían drogas modificadoras de la enfermedad (DME) y 25 pacientes $(22,3 \%)$ terapia biológica. 32 pacientes $(28,6 \%)$ cumplían criterios de Minimal Disease Activity (MDA) ${ }^{29}$. Las medidas de evaluación de los pacientes se presentan en la Tabla 1. 


\begin{tabular}{|l|c|}
\hline Variable & $n=112$ \\
\hline DAS28 $\boldsymbol{m}$ (RIC) & $3,38(2,58-4,31)$ \\
\hline IAS $\boldsymbol{m}$ (RIC) & $12,7(6,97-19,3)$ \\
\hline CDAI $\boldsymbol{m}$ (RIC) & $10(5,5-16)$ \\
\hline SDAI $\boldsymbol{m}$ (RIC) & $11,3(5,7-17)$ \\
\hline DAPSA $\boldsymbol{m}$ (RIC) & $14,3(7-22,4)$ \\
\hline HAQ-A $\boldsymbol{m}$ (RIC) & $\mathbf{0 , 7 5 ( 0 , 2 5 - 1 , 1 2 5 )}$ \\
\hline BASDAI $\boldsymbol{m}$ (RIC) & $4,27(1,9-6,7)$ \\
\hline Rigidez matinal (EVG cm) $\boldsymbol{m}$ (RIC) & $2(0-5)$ \\
\hline Dolor (EVG cm) $\boldsymbol{m}$ (RIC) & $5(2-7)$ \\
\hline Global del paciente (EVG cm) $\boldsymbol{m}$ (RIC) & $5(2-6)$ \\
\hline Global del médico (EVG cm) $\boldsymbol{m}$ (RIC) & $3(1-4,75)$ \\
\hline
\end{tabular}

DAS28: Disease Activity Score; IAS: Simplified Activity Index; CDAl: Clinical Disease Activity Index; SDAl: Simplified Disease Activity Index; DAPSA: Disease Activity for Psoriatic Arthritis; HAQ-A: Health Assessment Questionnaire, versión Argentina; BASDAl: Bath Ankylosing Spondylitis Disease Activity Index; EVG: Escala Visual Graduada $(0-10 \mathrm{~cm})$; RIC: Rango Intercuartilo.

Tabla 1. Medidas de evaluación en 112 pacientes con artritis psoriásica.

\begin{tabular}{|l|c|c|}
\hline \multirow{2}{*}{ Variable } & \multicolumn{2}{|c|}{ FATIGA } \\
\cline { 2 - 3 } BASDAI & $\mathbf{R h o}$ & $\mathbf{p}$ \\
\hline BASDAI pregunta N01 & $\mathbf{0 , 6 3}$ & $\mathbf{0 , 0 0 0 1}$ \\
\hline DAS 28 & $\mathbf{0 , 4 8}$ & $\mathbf{0 , 0 0 0 1}$ \\
\hline DAPSA & $\mathbf{0 , 4 5}$ & $\mathbf{0 , 0 0 0 1}$ \\
\hline HAQ-A & $\mathbf{0 , 5}$ & $\mathbf{0 , 0 0 0 1}$ \\
\hline PSAQoL & $\mathbf{0 , 4 2}$ & $\mathbf{0 , 0 0 0 1}$ \\
\hline ASQoL & $\mathbf{0 , 4 9}$ & $\mathbf{0 , 0 0 0 1}$ \\
\hline BASFI & $\mathbf{0 , 4 8}$ & $\mathbf{0 , 0 0 0 1}$ \\
\hline Dolor (EVG cm) & $\mathbf{0 , 4 7}$ & $\mathbf{0 , 0 0 0 1}$ \\
\hline Rigidez matinal (EVG cm) & $\mathbf{0 , 4 8}$ & $\mathbf{0 , 0 0 0 1}$ \\
\hline ERS (mm/hr) & $\mathbf{0 , 5 5}$ & $\mathbf{0 , 0 0 0 1}$ \\
\hline PCR (mg/dl) & 0,095 & 0,319 \\
\hline
\end{tabular}

BASDAl: Bath Ankylosing Spondylitis Disease Activity Index; DAS28: Disease Activity Index; DAPSA: Disease Activity for Psoriatic Arthritis; HAQ-A: Health Assessment Questionnaire, versión Argentina; PSAQoL: Psoriatic Arthritis Quality of Life; ASQoL: Ankylosing Spondylitis Quality of Life; BASFl: Bath Ankylosing Spondylitis Functional Index; EVG: Escala Visual Graduada (0-10cm); ERS: Eritrosedimentación; PCR: Proteína C-reactiva.

Tabla 2. Correlación de fatiga con otras medidas de evaluación.

La mediana de fatiga por EVG fue de $3 \mathrm{~cm}$ (RIC 0-6), y al considerar la pregunta número 1 del BASDAI relacionada a cansancio/fatiga la mediana fue $4 \mathrm{~cm}$ (RIC 2,5-7). La fatiga tuvo aceptable correlación con medidas de actividad de la enfermedad, calidad de vida y capacidad funcional, y no se correlacionó con los reactantes de fase aguda (Tabla 2).

Al comparar los pacientes con y sin fatiga, de acuerdo al punto de corte EVG $\geq 6 \mathrm{~cm}$, las medidas de evaluación fueron significativamente peores en los pacientes con fatiga, sin encontrarse diferencias en las características

\begin{tabular}{|c|c|c|c|}
\hline \multirow[b]{2}{*}{ Variable } & \multicolumn{3}{|c|}{ FATIGA $(E V G \geq 6 \mathrm{~cm})$} \\
\hline & Sí & No & $\mathbf{P}$ \\
\hline MASES X ( \pm ) DE & $2,69(2,7)$ & $1,21(1,69)$ & 0,01 \\
\hline DAS28 X ( \pm ) DE & $4,32(1,35)$ & $3,18(1,16)$ & 0,0001 \\
\hline CPDAI X ( \pm ) DE & $7,58(3,88)$ & $5,89(3,25)$ & 0,01 \\
\hline HAQ-A X (士) DE & $1,25(0,67)$ & $0,64(0,6)$ & 0,0001 \\
\hline BASFI X ( \pm ) DE & $5,58(2,58)$ & $2,85(2,75)$ & 0,001 \\
\hline PsAQoL X ( \pm ) DE & $11,22(4,49)$ & $5,78(5,86)$ & 0,0001 \\
\hline ASQoL X ( \pm ) DE & $11,06(4,88)$ & $5,66(5,6)$ & 0,0001 \\
\hline DAPSA X ( \pm ) DE & $25,88(15,81)$ & $12,22(9,12)$ & 0,0001 \\
\hline BASDAI X ( \pm ) DE & $6,68(2,06)$ & $3,32(2,52)$ & 0,0001 \\
\hline Edad X ( \pm$)$ DE & $52,91(11,62)$ & $53,3(13,33)$ & 0,88 \\
\hline Duración de la enfermedad $X( \pm) \mathrm{DE}$ & $9,37(7,75)$ & $11,91(8,31)$ & 0,14 \\
\hline Sexo $n^{\circ}(\%)$ & $31(28,6)$ & $79(71,4)$ & 0,4 \\
\hline
\end{tabular}

MASES: Maastricht Ankylosing Spondylitis Enthesitis Score; DAS28: Simplified Disease Activity; CPDAl: Composite Psoriatic Disease Activity Index; HAQ-A: Health Assessment Questionnaire, versión Argentina; BASFl: Bath Ankylosing Spondylitis Functional Index; PSAQoL: Psoriatic Arthritis Quality of Life; ASQoL: Ankylosing Spondylitis Quality of Life; DAPSA: Disease Activity for Psoriatic Arthritis; BASDAl: Bath Ankylosing Spondylitis Disease Activity Index; DE: Desvío Estándar; EVG: Escala Visual Graduada.

Tabla 3. Comparación entre pacientes con y sin fatiga categorizados según EVG de fatiga $\geq 6 \mathrm{~cm}$.

\begin{tabular}{|l|c|c|c|}
\hline Variable & Coeficiente $\beta$ & $\mathbf{t}$ & $\mathbf{p}$ \\
\hline DAPSA & $\mathbf{0 , 3 6 7}$ & $\mathbf{3 , 6 9 1}$ & $\mathbf{0 , 0 0 0 1}$ \\
\hline PsAQoL & $\mathbf{0 , 2 7 6}$ & $\mathbf{2 , 6 6 1}$ & $\mathbf{0 , 0 0 9}$ \\
\hline Sexo & 0,36 & 0,449 & 0,861 \\
\hline HAQ-A & 0,67 & 0,591 & 0,556 \\
\hline Tiempo de evolución & $-0,1$ & $-1,208$ & 0,23 \\
\hline Edad & 0,035 & 0,421 & 0,675 \\
\hline
\end{tabular}

Variable dependiente: Fatiga por EVG

DAPSA: Disease Activity for Psoriatic Arthritis; PSAQoL: Psoriatic Arthritis Quality of Life; HAQ-A: Health Assessment Questionnaire, versión Argentina; EVG: Escala Visual Graduada.

Tabla 4. Variables asociadas a fatiga. Regresión lineal múltiple.

sociodemográficas, como se muestra en la Tabla 3.

En el análisis de regresión lineal múltiple, utilizando la fatiga por EVG como variable dependiente, ajustando por sexo, edad y tiempo de evolución, la actividad de la enfermedad articular periférica (DAPSA coef $\beta: 0,36$, $\mathrm{p}=0,0001)$ y la calidad de vida (PsAQoL coef $\beta: 0,27$, $\mathrm{p}=0,009)$ se asociaron significativamente a fatiga (Tabla 4 ).

\section{Discusión}

La evaluación de fatiga en APs ha sido un dominio fuertemente recomendado para incluir en los ensayos $\operatorname{clínicos}^{30}$. En este estudio, aproximadamente el 30\% de nuestra cohorte de pacientes presentó EVG de fatiga $\geq 6$ $\mathrm{cm}$, lo que concuerda con estudios previos ${ }^{10}$. Nuestros 
resultados indican que la fatiga se asocia independientemente con la actividad de la enfermedad articular periférica y la calidad de vida. En otros estudios, la fatiga se asoció al dolor, sexo femenino, discapacidad funcional física y trastornos psicológicos asociados ${ }^{10}$, siendo también una barrera a la hora de llevar a cabo las actividades laborales ${ }^{31}$. Estos resultados pueden tener implicancias terapéuticas, ya que un adecuado control de la actividad de la enfermedad podría eventualmente mejorar la fatiga en estos pacientes y colaborar a un mejor control global de la enfermedad.

Las limitaciones de este estudio fueron: la evaluación de fatiga sólo se realizó a través de EVG sin utilizar algún cuestionario específico para medir esta variable como el FSS (Fatigue Severity Scale) o el FACIT (Functional Assessment of Chronic Illnes Therapy ${ }^{32}$. Estudios previos concluyen que la evaluación de la fatiga es insuficientemente cubierta por cuestionarios que evalúan calidad de vida o capacidad funcional ${ }^{9,10}$, sin embargo hemos reportado formalmente en un estudio previo que el FSS tiene una excelente correlación con la pregunta $\mathrm{N}^{\circ} 1$ del BASDAI en pacientes con Espondilitis Anquilosante $(\mathrm{EA})^{33}$. El punto de corte de fatiga medido por EVG en $6 \mathrm{~cm}$ se definió de manera arbitraria. Otra limitante fue no haber evaluado específicamente otras variables que pueden influir sobre la fatiga como la depresión, ansiedad y calidad del sueño, considerando que estudios previos han demostrado que la depresión es uno de los principales factores asociados ${ }^{32}$.

En conclusión, la fatiga es un síntoma frecuente en APs y se asocia independientemente con actividad de la enfermedad articular periférica y calidad de vida.

\section{Bibliografía}

1. Arturi A. Diagnóstico en Reumatología y Enfermedades Autoinmunes Sistémicas. Edición Abbvie. Capítulo 8. Pág. 197-202. 2014.

2. Maldonado Ficco H, Citera G, Maldonado Cocco JA. Prevalence of psoriatic arthritis in psoriasis patients according to newer classification criteria. Clin Rheumatol 2014;33(2):243-6.

3. Maldonado Cocco J, Citera G. Reumatología. Segunda Edición, Ediciones Azzurras. Capítulo 33. Pág 462-70. 2012.

4. Castelli A, Maisterrena CE, Kerzberg EM. Calidad de vida en fibromialgia y artritis psoriática. Revista Latinoamericana de Psoriasis y Artritis Psoriásica 2012;5:26-33.
5. Hustead JA, Thavaneswaran A, Chandran V, Gladman D. Incremental Effects of Comorbidity on Quality of Life in Patients with Psoriatic Arthritis. J Rheumatol 2013;40(8):1349-56.

6. Fernández Sueiro JL, Lema Gontad JM. Factores pronósticos en la artritis psoriásica. Clin Reumatol 2012; 8 (Supl 1):57-9.

7. Zink A, Thiele K, Huscher D, Listing J, Sieper J, Krause A, et al. Healthcare and burden of disease in psoriatic arthritis. A comparison with rheumatoid arthritis and ankylosing spondylitis. J Rheumatol 2006;33(1):86-90.

8. Rosen CF, Mussani F, Chandran V, Eder L, Thavaneswaran A, Gladman D. Patients with psoriatic arthritis have worse quality of life than those with psoriasis alone. Rheumatology 2012;51(3):571-6

9. Husted JA, Tom BD, Farewell VT, Gladman D. Longitudinal Analysis of Fatigue in Psoriatic Arthritis. J Rheumatol 2010; 37 (9):1878-84.

10. Husted JA, Tom BD, Schentag CT, Farewell VT, Gladman D. Occurrence and correlates of fatigue in psoriatic arthritis. Ann Rheum Dis 2009;68(10):1553-8.

11. Taylor W, Gladman D, Helliwell P, Marchesoni A, Mease P, Mielants H. Classification criteria for psoriatic arthritis: development of new criteria from a large international study. Arthritis Rheum 2006;54(8):266573.

12. Wolfe F, Smythe HA, Yunus MB, Bennett RM, Bombardier C, Goldenberg DL, Tugwell P, Campbell SM, Abeles M, Clark P, et al. The American College of Rheumatology 1990 Criteria for the Classification of Fibromyalgia. Report of the Multicenter Criteria Committee. Arthritis Rheum 1990 ;33(2):160-72.

13. Heuft-Dorenbosch L, Spoorenberg A, van Tubergen A, Landewe $\mathrm{R}$, van ver Tempel $\mathrm{H}$, Mielants $\mathrm{H}$, et al. Assessment of enthesitis in ankylosing spondylitis. Ann Rheum Dis 2003;62(2):127-32.

14. Marks R, Barton SP, Shuttleworth D, Finlay AY. Assessment of disease progression in psoriasis. Arch Dermatol 1989;125 (2):235-40.

15. Williamson L, Dalbeth N, Dockerty JL, Gee BC, Weatherall R, Wordsworth BP. Extended report: nail disease in psoriatic arthritis--clinically important, potentially treatable and often overlooked. Rheumatology (Oxford) 2004;43(6):790-4.

16. Jenkinson TR, Mallorie PA, Whitelock HC, Kennedy LG, Garrett SL, Calin A. Defining spinal mobility in ankylosing spondylitis (AS). The Bath AS Metrology Index. J Rheumatol 1994;21(9):1694-8. 
17. Doward LC, Spoorenberg A, Cook SA, Whaley D, Helliwell PS, Kay LJ, et al. Development of the ASQoL: a quality of life instrument specific to ankylosing spondylitis. Ann Rheum Dis 2003;62(1):20-6.

18. McKenna SP, Doward LC, Whalley D, Tennant A, Emery P, Veale DJ. Development of the PsAQoL: a quality of life instrument specific to psoriatic arthritis. Ann Rheum Dis 2004;63(2):162-9.

19. Finlay AY, Khan GK. Dermatology Life Quality Index (DLQI): a simple practical measure for routine clinical use. Clin Exp Dermatol 1994;19(3):210-6.

20. Citera G, Arriola MS, Maldonado Cocco JA. Validation and crosscultural adaptation of an Argentine Spanish version of the health assessment questionnaire disability index. J Clin Rheumatol 2004;10(3):110-5.

21. Calin A, Garrett SL, Whitelock H, Kennedy LG, O'Hea J, Mallorie P, et al. A new approach to functional ability in ankylosing spondylitis. J Rheumatol 1994;21(12):2281-5.

22. Garrett S, Jenkinson T, Kennedy LG, Whitelock H, Gaisford P, Calin A. A new approach to defining disease status in ankylosing spondylitis: the Bath Ankylosing Spondylitis Disease Activity Index. J Rheumatol 1994;21(12):2286-91.

23. Felson DT, Anderson JJ, Boers M, Bombardier C, Chernoff M, Fried B, et al The American College of Rheumatology preliminary core set of disease activity measures for rheumatoid arthritis clinical trials. The Committee on Outcome Measures in Rheumatoid Arthritis Clinical Trials. Arthritis Rheum 1993;36(6):729-40.

24. Schoels M, Aletaha D, Funovits J, Kavanaugh A, Baker D, Smolen JS. Application of the DAREA/DAPSA score for assessment of disease activity in psoriatic arthritis. Ann Rheum Dis 2010;69(8):1441-7.

25. Mumtaz A, Gallagher P, Kirby B, Waxman R, Coates LC, Veale JD, et al. Development of a preliminary composite disease activity index in psoriatic arthritis. Ann Rheum Dis 2011;70(2):272-7.

26. Smolen JS, Breedveld FC, Schiff MH, Kalden JR, Emery P, Eberl G, et al. A simplified disease activity index for rheumatoid arthritis for use in clinical practice. Rheumatolology (Oxford) 2003;42(2):244-57.

27. Aletaha D, Nell VPK, Stamm T, Uffmann M, Pflugbeil S, Machold K, et al. Acute phase reactants add little to composite disease activity indices for rheumatoid arthritis: validation of a clinical activity score. Arthritis Res Ther 2005;7(4):796-806.
28. Curet AV, Rillo OL, Chaparro del Moral RE, Papasidero SB, Citera G, Maldonado Cocco JA, et al. Modificación y aplicación de un índice de actividad simplificado (IAS) en pacientes con artritis reumatoidea [abstract]. Rev Argent Reumatol 2005; 16(Supl 1):13.

29. Coates LC, Fransen J, Helliwell PS. Defining minimal disease activity in psoriatic arthritis: a proposed objective target for treatment. Ann Rheum Disease 2010;69(1):48-53.

30. Kwok T, Pope JE. Minimally Important Difference for Patient-reported Outcomes in Psoriatic Arthritis: Health Assessment Questionnaire and Pain, Fatigue, and Global Visual Analog Scales. J Rheumatol 2010;37 (5):1024-8.

31. Wallenius M, Skomsvoll JF, Koldingsnes W, Rødevand E, Mikkelsen K, Kaufmann C, et al. Work disability and health-related quality of life in males and females with psoriatic arthritis. Ann Rheum Dis 2009;68(5):685-9.

32. Minnock P, Kirwan J, Veale D, FitzGerald O, Bresnihan B. Fatigue is an independent outcome measure and is sensitive to change in patients with psoriatic arthritis. Clin Exp Reumatol 2010;28(3):401-4.

33. Schneeberger EE, Marengo MF, Dal Pra F, Maldonado Cocco JA, Citera G. Fatigue assessment and its impact in the quality of life of patients with ankylosing spondylitis. Clin Rheumatol 2015;34(3):497-501. 